[When citing this chapter, refer to Behaviour 152 (2015) 259-283]

\title{
Relationship quality in captive bonobo groups
}

\author{
Jeroen M.G. Stevens ${ }^{\mathrm{a}, *}$, Evelien de Groot ${ }^{\mathrm{a}}$ and Nicky Staes ${ }^{\mathrm{a}, \mathrm{b}}$ \\ ${ }^{a}$ Centre for Research and Conservation, Royal Zoological Society of Antwerp, \\ Koningin Astridplein 20-26, 2018 Antwerp, Belgium \\ ${ }^{\mathrm{b}}$ Department of Biology, University of Antwerp, Groenenborgerlaan 171, \\ 2020 Antwerp, Belgium \\ *Corresponding author's e-mail address: jeroen.stevens@kmda.org
}

Accepted 4 January 2015; published online 28 January 2015

\begin{abstract}
We use Principal Component Analyses (PCA) to describe components of social relationship quality in bonobos. We find a three component structure, with the first two components, labelled Value and Compatibility, closely matching the theoretical constructs as well as components reported for chimpanzees and other primates. The third component differed but was abandoned based on Parallel Analysis. Among bonobos, female-female dyads have higher Value and Compatibility. Relationships between males are characterised by low Value and Compatibility. Dyads that had been housed together for a longer time and maternally related ones also have more valuable relationships, while individuals close in rank have low compatibility. The results confirm the strong bonds among female bonobos, but for the first time can describe how they differ qualitatively from close bonds reported for captive chimpanzee females. We suggest future studies should also include Parallel Analysis to more accurately describe the number of components in relationship quality.
\end{abstract}

\section{Keywords}

social organisation, Pan paniscus, relationship quality.

\section{Introduction}

Living in social groups can bring about fitness benefits (Kappeler \& van Schaik, 2002). Within social groups not all relationships are equal, and individuals of many species are known to have diversified social relationships, resulting in complex social networks. Social relationships can be beneficial for individual fitness, and recent studies have shown that strong friendships between females can result in higher longevity, fecundity and offspring survival (Silk et al., 2003, 2009, 2010; Silk, 2007; Cameron et al., 2009; Frère 
et al., 2010). Similarly, males can increase their fitness by forming bonds or friendships with other males (Feh, 1999; Schülke et al., 2010; Wiszniewski et al., 2012; Gilby et al., 2013). Finally, there is accumulating evidence that male-female bonds can also increase the fitness of males in baboons, macaques and chimpanzees (Moscovice et al., 2010; Massen et al., 2012; Langergraber et al., 2013).

While the influence of high quality social relationships on individual fitness is becoming increasingly clear, describing and measuring the quality of these social bonds is still under much debate. Hinde (1976) and Kummer (1978) describe social relationships as an emergent property of repeated social interactions between two individuals and can be seen as investments, where individuals are expected to invest more in valuable partners. Following these concepts, Cords \& Aureli (2000) proposed that relationship quality could be described by three components, labelled Value, Compatibility and Security. Value refers to the direct benefits gained by the relationship, such as food sharing or agonistic support. Compatibility is measured by tolerance and affiliation, based on a history of shared history of social exchanges. Security means that the behaviour of partners is predictable and consistent over time. Subsequently, several studies have sought to specify these relationship components and relate them to other behaviours, conciliatory tendencies for example. However chosen operational measures of each component often differ between studies (reviewed in Cords \& Aureli, 2000 and Fraser et al., 2008). Traditionally, behavioural ecologists have looked at the frequency of one or a few behaviours, such as proximity, grooming or support, to measure relationship value (e.g., Lehman \& Boesch, 2009; Langergraber et al., 2012; Massen et al., 2012); alternatively, they inferred closeness of social relationships indirectly, for example by assuming that kin have higher quality relationships than non-kin, or that members of the dispersing sex have lower relationship quality than dyads that are philopatric. The latter assumption may be problematic (see below). More recently, principal component analysis (PCA) has been used to describe relationship quality in captive chimpanzees (Fraser et al., 2008). The benefit of Principal Component Analyses is that behavioural variables can be objectively reduced to a few behavioural dimensions, which are comprehensive and conceptually more coherent and can then be related to different variables. In a study on zoo housed chimpanzees, Fraser et al. (2008) found three components of relationship quality 
from nine behavioural variables, which matched to the three theoretical constructs that had been proposed. Once these dimensions of relationship quality have been assessed, it is then possible to look at variation across different dyads. Fraser et al. (2008) found that value was higher among related dyads, between chimpanzees that had been housed together longer or where age differences were smaller. Compatibility was higher among female-female than among male-male or male-female dyads, and was also higher among male-female compared to male-male dyads. Furthermore, this component was higher for related chimpanzees, and among individuals that had been housed together longer. Finally, security again was higher in related dyads, among male-female dyads and dyads of similar age, but was lower in dyads that had been housed together longer. Thus, this first study could clearly identify components of relationship quality and show that certain preconceptions about the influence of kin and sex-combination were not present for this captive group of chimpanzees. Subsequent studies have used the same approach and a three-component model has been found in a different group of chimpanzees (Koski et al., 2012), in Japanese macaques (Majolo et al., 2010), Barbary macaques (McFarland \& Majolo, 2011) as well as in ravens (Fraser \& Bugnyar, 2010). In wild spider monkeys the PCA resulted in only two components, showing the need for a wider taxonomic angle (Rebecchini et al., 2011). Moreover, it has been noted that while these models use dyadic values, relationships within dyads can be asymmetric (Majolo et al., 2010). All these studies seem to indicate that multiple components can be described, but more studies are needed to see how consistent they are across taxa.

While two studies have investigated relationship quality in captive chimpanzees, much less is known about relationship quality in the chimpanzees' sibling species, the bonobo (Pan paniscus). Quantifying the relationship values can shed light on the ongoing debate about how different these two species really are, and can provide more insight on the influence of kinship in social bonding. Traditionally, differences between these two sibling species have been highlighted. Both species live in societies that are comparable in size, and in both species females migrate to neighbouring communities when they reach adolescence (Goodall, 1986; Kano, 1992; Furuichi et al., 1998; Stumpf, 2007). However, chimpanzee society is described as male bonded and females are relatively more solitary (Goodall, 1986; Stumpf, 2007) while bonobo society is female-centred with strong relationships among females, between females and their adult sons, and perhaps even between females and 
unrelated males (Badrian \& Badrian, 1984; White, 1988; Furuichi, 1989, 2011; Parish, 1996; Hohmann \& Fruth, 2002; Stevens et al., 2006). These close bonds between female bonobos have received much attention, as they challenge assumptions about socio-ecological models and kin selection that predict closer bonds between related individuals, and even about our own evolutionary past (Parish \& de Waal, 2000).

However, the chimpanzee-bonobo dichotomy in terms of social bonding has been questioned on two levels. Firstly, the idea of chimpanzees as being only male bonded has been challenged. Chimpanzee males do indeed form meaningful relationships with other males (Mitani, 2009; Gilby et al., 2013), but so do female chimpanzees in the wild and in captivity (Baker \& Smuts, 1994; Langergraber et al., 2009; Lehman \& Boesch, 2009). Furthermore, kinship did not predict bonding in wild male and female chimpanzees (Langergraber et al., 2007, 2009). Recently, long-term male-female relationships have been shown to influence male reproductive success in chimpanzees in East-Africa (Langergraber et al., 2013). Secondly, the strong female bonding of bonobos has been questioned. Previous research in captivity has shown very strong bonds between female bonobos (Parish, 1996), but often these females were closely related and in groups containing only one or two males that were always unrelated to the females. Therefore, the existence of female bonding in bonobos has been questioned and it has been proposed as a side effect of life in captivity (Stanford, 1998; Franz, 1999; Hohmann et al., 1999). It has been suggested that relationships between male and female bonobos are more important, even when controlling for kinship effects (Hohmann et al., 1999; Hohmann \& Fruth, 2002). Our own subsequent research on a larger dataset of several groups of zoo housed bonobos, in which females were generally unrelated and mother-son dyads were present, showed that grooming, proximity and proximity maintenance was not stronger among females than among females and unrelated males or among males. We found that only coalitionary support was consistently significantly more common among females than among males or among females and unrelated males (Stevens et al., 2006). However, the significance of these behavioural variables and their interdependence, in terms of the three proposed components of relationship quality, was not examined. Jaeggi et al. (2010) compared relationships in chimpanzees and bonobos, using a PCA on three behavioural variables (grooming frequency, proximity and support given). In the chimpanzees this resulted in one component of relationship 
quality, which had high loadings on grooming given, support and proximity. In the bonobos however, the data split into two components; the first was labelled 'affiliative relationship' and included a high loading of grooming and proximity and a negative loading of support. The second component was labelled 'political relationship' and had a high loading of support, minor loading of proximity and negative loading of grooming. This suggests that relationship quality in bonobos may differ from chimpanzees. However, the analyses of Jaeggi et al. (2010) were based on one group of six bonobos, studied for $125 \mathrm{~h}$, and were based on a limited set of behavioural variables. Therefore, a study that is based on a larger sample size and is more comparable to the chimpanzee studies (Fraser et al., 2008; Koski et al., 2012) is needed.

Here we use a larger set of data from captive bonobo groups, not including the data from Jaeggi et al. (2012) group, and include eight behavioural variables, resembling those used in the chimpanzee studies (Fraser et al., 2008; Koski et al., 2012). Our first aim is to describe components of Relationship Quality among captive bonobos, using Principal Component Analyses. We predict that Relationship Quality can be described in similar components to those found in chimpanzees. Second, we test which components are stronger in female-female dyads to further clarify the nature of female bonding in bonobos. Third, we look at other variables that have been found to affect the components of Relationship Quality in other species: kinship, tenure of the relationship, age difference and rank difference.

\section{Material and methods}

\subsection{Study groups}

We studied six groups of bonobos housed in four European Zoos (Wuppertal, Apenheul, Twycross, Planckendael, see Table 1). Data were only collected from individuals above seven years old, which is the earliest age of reproduction in captivity for male and female bonobos (Pereboom et al., 2011). In each group, the bonobos were housed together for most of the day and night and were only separated briefly during maintenance or cleaning work. Observations ran from the morning (usually around $8 \mathrm{~h} 30 \mathrm{~min}$ ) until dusk, when bonobos started building nests for the night (usually around 5.30 p.m., depending on season). The group in Planckendael was studied three times (in 1999, 2001 and 2011), however the group composition differed substantially between each period, and only a few dyads were present in more than one 
Table 1.

Composition and observation time for each of the five study groups.

\begin{tabular}{lcccc}
\hline $\begin{array}{l}\text { Group and year } \\
\text { of study }\end{array}$ & $\begin{array}{c}N \text { of males }> \\
7 \text { years }\end{array}$ & $\begin{array}{c}N \text { of females }> \\
7 \text { years }\end{array}$ & $\begin{array}{c}N \text { of immatures }< \\
7 \text { years }\end{array}$ & $\begin{array}{c}\text { Observation } \\
\text { time (h) }\end{array}$ \\
\hline Planckendael 1999 & 3 & 4 & 4 & 203 \\
Planckendael 2001 & 3 & 3 & 2 & 503 \\
Planckendael 2011 & 3 & 3 & 2 & 295 \\
Wuppertal 1999 & 4 & 2 & 1 & 193 \\
Apenheul 2001 & 3 & 5 & 3 & 493 \\
Twycross 2001 & 3 & 3 & 2 & 490 \\
\hline
\end{tabular}

Immatures were not included in any of the analyses.

group setting (overlap between period Planckendael 1999 and Planckendael 2001: 6 out of 15 dyads; overlap period Planckendael 2001 and Planckendael 2010: 1 out of 15 dyads; there was no further overlap in dyads between any of the other groups studied). When a dyad occurred in more than one study period, we chose the data for the dyad based on the period with the longest observation time. This resulted in a total sample of 102 different dyads composed of 17 females and 16 males. Each individual was represented in $6.18 \pm 0.23$ dyads (mean \pm SE). Data on Planckendael in 2011 were collected by EDG, all other data were collected by JMGS, following a standardised ethogram for social behaviour in bonobos.

\subsection{Data collection and analyses}

We used behaviour sampling to score social behaviours among individuals that were older than seven years of age. All occurrences of aggression, grooming, coalitionary support and peering were recorded. In each grooming bout, the participation of each partner was scored once. Subsequent switches between the active and passive role where not counted as new bouts (Vervaecke et al., 2000a) and frequency of grooming bouts within a dyad was calculated. Support was defined as all instances where an individual A intervenes with an aggression within $30 \mathrm{~s}$ in an agonistic interaction between two other individuals B and C to aid in attack or in defence (de Waal, 1978). We only studied triadic interventions because in polyadic interactions, where more than three individuals were involved, it was often unclear to whom the support was directed. When more individuals joined in support, these interactions were not considered. All aggressions from individual $\mathrm{C}$ towards $\mathrm{B}$ were considered as opportunities for support, regardless of the reaction 
of the victim B. We then corrected for the opportunity for individual A to provide support to $\mathrm{B}$ in a conflict with $\mathrm{C}$, by dividing the total number of supports between $\mathrm{A}$ and $\mathrm{B}$ by the total number of conflicts that individuals A or B had with other group members, excluding the conflicts they had with each other, and then multiplied this number by 100 (Vervaecke et al., 2000b; Stevens et al., 2006). Every support pro for an individual implied a contra support against a victim (de Waal, 1978). Here too, we corrected for the number of conflicts. Thus the target of a coalition is considered as the receiver of 'counter-intervention' (or contrasupport, sensu Vervaecke et al., 2000b). Proximity was scored using scan sampling, noting every fifteen minutes which individuals were within arm's reach (or less than one meter) from each other. For analyses, seven data points were chosen at random per observation day, to avoid interdependency of these data (Martin \& Bateson, 1993; Stevens et al., 2006). To calculate a proportional value the total number of randomised samples was then divided by the total number of samples taken per group.

For each dyad we used eight behavioural variables to include in the first Principal Component Analysis (PCA1; Table 2). We chose behavioural vari-

Table 2.

Behavioural variables entered in the Principal Component Analysis.

\begin{tabular}{llc}
\hline Behavioural variable & Definition & Mean \pm SE per dyad \\
\hline Grooming frequency & $\begin{array}{l}\text { Number of grooming bouts exchanged } \\
\text { within a dyad (i.e. the sum of all bouts } \\
\text { from A to B and from B to A) }\end{array}$ & $0.28 \pm 0.03$ \\
Grooming symmetry & $\begin{array}{l}\text { Symmetry of grooming within a dyad } \\
\text { (see text) }\end{array}$ & $0.26 \pm 0.02$ \\
Proximity & $\begin{array}{l}\text { Proportion of scans spent within arm's } \\
\text { reach }\end{array}$ & $0.12 \pm 0.07$ \\
Aggression frequency & $\begin{array}{l}\text { Frequency of all aggressive } \\
\text { interactions within a dyad }\end{array}$ & $0.17 \pm 0.02$ \\
Aggression symmetry & $\begin{array}{l}\text { Symmetry of aggression within a dyad } \\
\text { (see text) }\end{array}$ & $0.20 \pm 0.18$ \\
Support & $\begin{array}{l}\text { Index of agonistic support (frequency } \\
\text { of support/opportunity to support) } \\
\text { Index of counter-intervention } \\
\text { (frequency of counter-intervention/ }\end{array}$ & $0.48 \pm 0.08$ \\
Counter-intervention & $\begin{array}{l}\text { Fpportunity to intervene) } \\
\text { Frequency of peering }\end{array}$ & $0.46 \pm 0.07$ \\
\hline
\end{tabular}


ables that closely matched the studies on chimpanzees by Fraser et al. (2008) and Koski et al. (2012). To allow intergroup comparison with observation times that differed between groups, we transformed all behaviours either to frequencies by dividing by the number of group observation hours, or to proportions (see below). For frequencies of grooming and aggression between individuals $\mathrm{A}$ and $\mathrm{B}$, we added the total number of aggressions and grooming bouts from $\mathrm{A}$ to $\mathrm{B}$ and from $\mathrm{B}$ to $\mathrm{A}$, and divided by the total group observation time. Following Fraser et al. (2008), we calculated symmetry in grooming between individuals $A$ and $B$ using the following formula: A grooms B/(A grooms B + B grooms A). For each dyad, the lowest of the two values obtained reversing A's and B's roles was chosen to represent the degree of symmetry, so values ranged from 0 to 0.5 , with higher values indicating more symmetrical exchanges. Symmetry in aggression was calculated in the same way. Food sharing was not consistently scored in all of the study groups; therefore, successful begging could not be included as a variable. Instead, peering behaviour (i.e., "the actor stares at the receiver's face from very close distance, up to a few centimetres" Kano, 1992, p. 200) was used as a proxy for tolerance. The function of peering in bonobos is poorly understood, but according to some it is a begging gesture (Kano, 1980), while others have stated it is "a solicitation for initiating affiliative interactions" (Idani, 1995) or 'a request for social tolerance' (Stevens et al., 2005a). Consistency of affiliation could not be measured in our study, since observation periods lasted between one to three months. Unlike the more conspicuous behaviours, approaches and their response could not reliably be scored using the all occurrence sampling and were therefore not included in the analyses.

We analysed the variables with a first principal component analysis (PCA1) with varimax rotation and Kaiser normalization. To improve normality, we transformed the data using square root transformation on all frequency data (grooming, aggression, support, counter-intervention, peering). Kaiser's measure of sampling adequacy was 0.65 , which is on the low side, but acceptable (Budaev, 2010). To determine the number of components, we first followed Kaiser's rule and accepted all components which had an eigenvalue greater than 1 (Kaiser, 1960), in combination with visual inspection of the scree plot (Cattell, 1966). Coefficients of correlation greater than 0.5 or less than -0.5 were considered to be high loadings. Although Kaiser's rules and/or investigation of scree plots have also been used as criteria in all previous studies investigating relationship quality to determine 
the number of components (Fraser et al., 2008; Fraser \& Bugnyar, 2010; Majolo et al., 2010; McFarland \& Majolo, 2011; Rebecchini et al., 2011; Koski et al., 2012), this procedure may lead to overestimating the number of factors, and the use of Parallel Analysis has been shown to be more reliable to determine the number of extracted components (Horn, 1965; Franklin et al., 1995; O'Connor, 2000). Parallel Analysis is a "sample based adaptation of the population based Kaiser's rule" (Zwick \& Velicer, 1986) and allows the researchers to determine the significance of components (Franklin et al., 1995). Only when eigenvalues from PCA are larger than the corresponding eigenvalues from Parallel Analysis, should the components be considered as true components. We therefore used SAS to conduct Parallel Analysis to confirm the number of extracted factors (O'Connor, 2000). When the Parallel Analysis indicated only two out of the three original components should be contained in the final analysis, we performed a second Principal Component Analysis extracting just two components to determine the variable loadings per component and individual component scores (PCA2).

Next, we used Linear Mixed Models to investigate the influence of kinship (separate factor for maternal and paternal kin), tenure (in years), sex combination (female-female; male-female; male-male), rank difference, and age difference (in years) on the extracted scores from PCA2. Kinship information was derived from the studbook; within each group all paternities were known (Pereboom et al., 2011). We only included kin with relatedness coefficients larger than 0.125 and treated it as a binary variable (kin or non-kin). For the factor 'maternal kin', this included one mother-daughter pair, six mother-son pairs and three maternal half-sibling pairs; for paternal kinship this included five father-son pairs and three paternal half-sibling pairs. Since females usually are transferred between zoos when they reach adolescence, our study groups contained no father-daughter pairs. There were also no full siblings. Relationship tenure was calculated from the studbook and included the number of years two individuals had spent together (with an error margin of 0.5 years; minimum 0.5 , maximum 22 years). Rank differences were taken from Stevens et al. (2007) for five of the groups and calculated in the same way for Planckendael 2010 by constructing an ordinal dominance hierarchy based on the analyses of 'fleeing upon aggression' (Vervaecke et al., 2000a), and calculating net differences between individuals' ordinal ranks using MATMAN software (de Vries et al., 1993). Age difference was calculated by taking the differences of (estimated) years of birth (taken from 
Pereboom et al., 2011) for the two members of a dyad. Because of the relatively low number of related individuals in our groups (which were mostly mother-son dyads), we could not include interaction factors for sex combination, kin or age in the linear models. To account for interdependence of the data, we included the identities of the two bonobos forming each dyad, as well as the factor 'group' as random variables in the statistical model. We used $F$-tests and backward selection to construct a final model that only retained variables with significant effects. When a significant effect was found for categorical variables, we performed post-hoc pairwise comparisons, using Tukey tests for multiple comparisons of means. PCA, Parallel Analysis, and linear mixed models were conducted using SAS software (version 9.0) with the critical $\alpha$ set at 0.05 .

\section{Results}

\subsection{Components of relationship quality}

Using Kaiser's criterion, three components were extracted from PCA1, explaining $31.03 \%, 17.57 \%$ and $13.64 \%$ of overall variance respectively, totalling $62.24 \%$. The first component had high positive loadings for proximity, grooming frequency, support and peering. These variables are very close to the definition of relationship value by other studies (Cords \& Aureli, 2000; Fraser et al., 2008) and thus could be labelled 'Value'. The second component included high values for aggression frequency and high values for aggression symmetry (indicating asymmetric aggression) variables that suggest incompatibility (Fraser et al., 2008). In the further analyses, we reversed the signs for the scores obtained for each dyad for that component so that the values represented the degree of compatibility as opposed to the incompatibility of the dyad (cf. Fraser et al., 2008). The third component included a high positive loading for counter-intervention and a negative loading for grooming symmetry. Since grooming symmetry loaded high and positive on Fraser et al. (2008)'s security component, our component seems to measure insecurity. However, subsequent Parallel Analysis showed that only the two first components had higher eigenvalues than randomly generated values (Figure 1). Therefore, the third factor should not be considered further, and was dropped from further analyses. The factor loadings for the two-component model, resulting from PCA2, are shown in Table 3. In this 


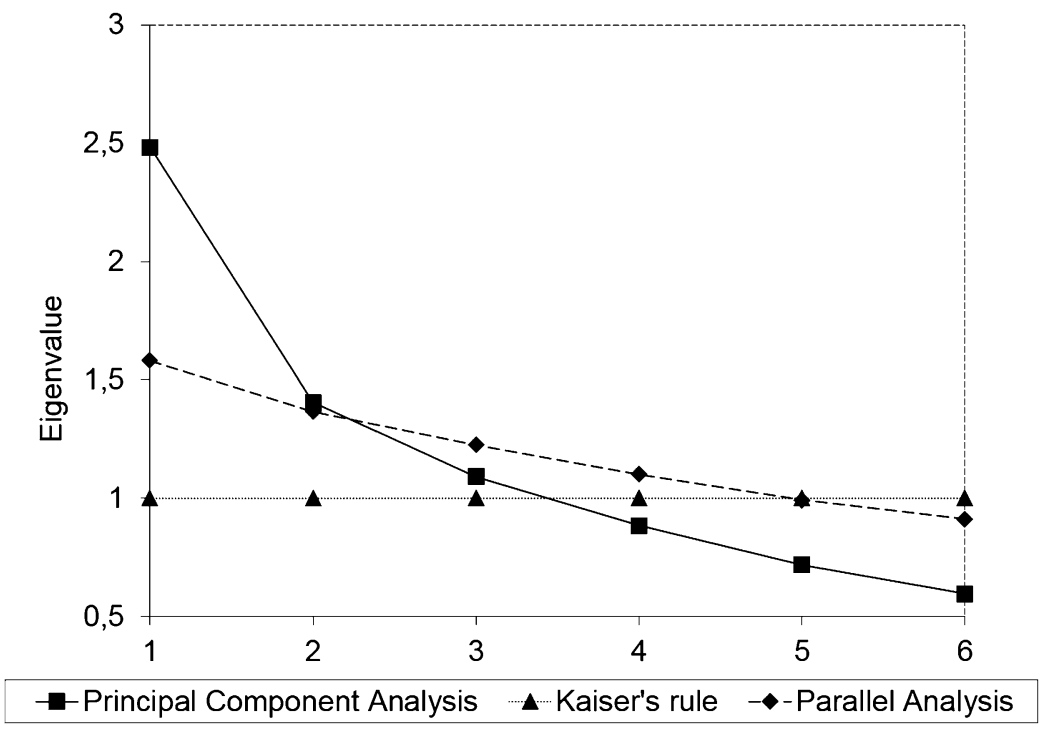

Figure 1. Comparison of eigenvalues of the components extracted in the Principal Component Analysis with Kaiser's criterion and with eigenvalues exctracted based on Parallel Analysis. Based on Kaiser's rule, three components would be retained. Parallel Analysis shows that the third component is not valid.

model, the first component explained $34.80 \%$ of total variance, and contained high positive loadings for proximity, grooming, support, peering and grooming reciprocity, and therefore corresponds to the theoretical component 'Value'. The second component explained $19.25 \%$ of total variance and contained high positive loadings for aggression frequency and aggression reciprocity and we therefore labelled it 'Compatibility'. The behavioural variable counter-intervention did not load high on either of the two components and therefore was abandoned in the final model.

\subsection{Factors affecting relationship quality}

The first component, labelled Value, was significantly influenced by the following factors: sex combination, age difference, maternal kinship and tenure (Table 4). Post-hoc comparisons showed that female-female dyads had significantly higher value than male-female $\left(p_{\text {adj }}<0.0001\right)$ and male-male dyads ( $\left.p_{\text {adj }}<0.0001\right)$, and male-female dyads had significantly higher values than male-male dyads $\left(p_{\text {adj }}=0.0013\right.$ ) (Figure 2). Maternal kin had significantly higher value than non-kin, but there was no effect of paternal 


\section{Table 3.}

Varimax rotated component matrix of the final Principal Component Analyses (PCA2) run on the dyadic scores for 8 variables.

\begin{tabular}{lcc}
\hline Variable & Value & (in)Compatibility \\
\hline Proximity & $0.88^{*}$ & 0.09 \\
Groom frequency & $0.72^{*}$ & 0.28 \\
Support & $0.66^{*}$ & -0.28 \\
Peering & $0.61^{*}$ & -0.07 \\
Groom symmetry & $0.55^{*}$ & -0.17 \\
Aggression symmetry & 0.09 & $0.76^{*}$ \\
Aggression frequency & 0.00 & $0.67^{*}$ \\
Counter-intervention & -0.25 & 0.44 \\
\% of variation explained & 34.80 & 19.25 \\
Eigenvalue & 2.44 & 1.35 \\
\hline
\end{tabular}

An asterisk indicates high loadings ( $>0.5$ or $<-0.5)$.

Table 4.

Effects of the variables on the components Value and Compatibility.

\begin{tabular}{lcrc}
\hline Component & $B \pm \mathrm{SE}$ & $t$ value & $p$ \\
\hline Value & & & \\
Intercept & $-0.38 \pm 0.38$ & -1.00 & 0.32 \\
Sex combination (MM vs FF) & $1.55 \pm 0.25$ & 6.21 & $<0.0001$ \\
Sex combination (MM vs MF) & $0.84 \pm 0.20$ & 4.31 & $<0.0001$ \\
Maternal kin & $-0.86 \pm 0.26$ & -3.32 & 0.001 \\
Tenure & $0.054 \pm 0.017$ & 3.16 & 0.003 \\
Age difference & $0.017 \pm 0.013$ & 1.31 & 0.19 \\
Rank difference & $0.002 \pm 0.051$ & 0.04 & 0.97 \\
Paternal kin & $-0.27 \pm 0.39$ & -0.70 & 0.49 \\
Compatibility & & & \\
Intercept & $0.50 \pm 0.29$ & 1.70 & 0.10 \\
Sex combination (MM vs FF) & $0.79 \pm 0.27$ & 2.90 & 0.005 \\
Sex combination (MM vs MF) & $-0.058 \pm 0.22$ & -0.26 & 0.80 \\
Rank difference & $0.11 \pm 0.06$ & 1.95 & 0.05 \\
Maternal kin & $0.47 \pm 0.29$ & 1.60 & 0.11 \\
Paternal kin & $0.60 \pm 0.42$ & 1.44 & 0.15 \\
Tenure & $0.002 \pm 0.018$ & 0.10 & 0.91 \\
Age difference & $0.004 \pm 0.016$ & 0.24 & 0.80 \\
\hline
\end{tabular}




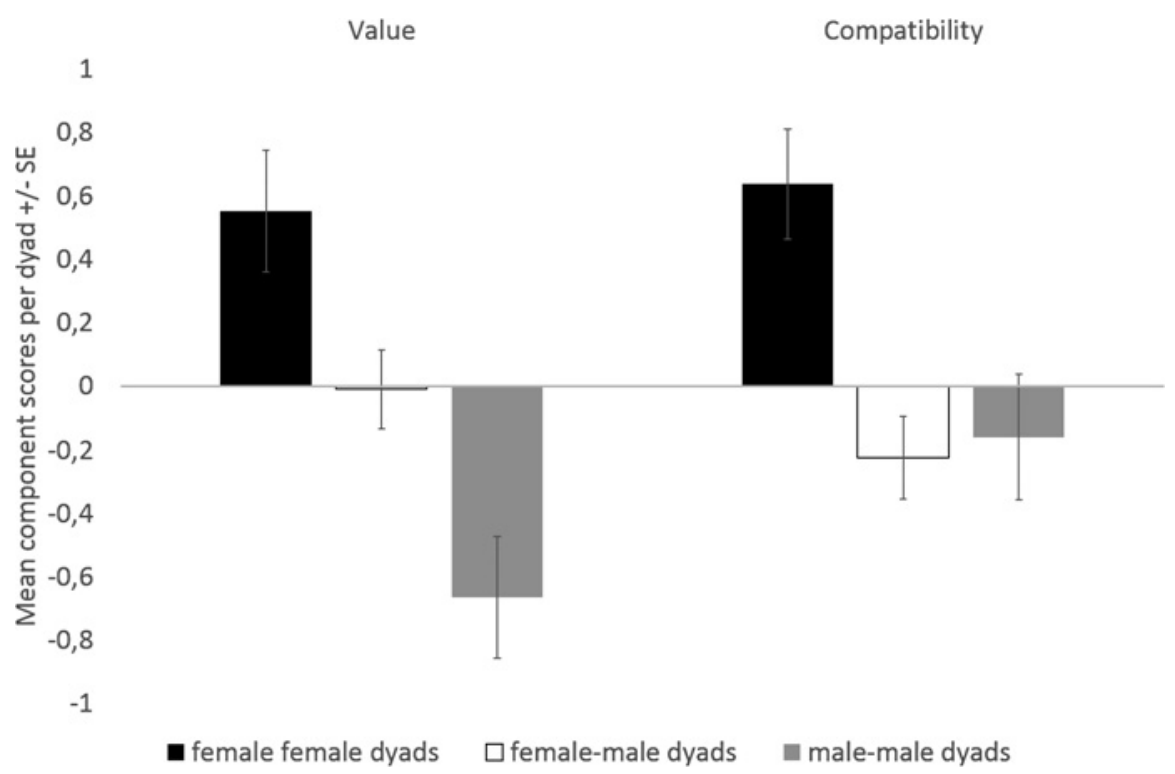

Figure 2. Mean relationship quality (PCA component scores) of female-female, female-male and male-male dyads.

kin. Dyads that had longer tenure had higher value, and value increased with age difference.

Compatibility was influenced by sex combination and rank difference. Female-female dyads had higher compatibility than male-female dyads $\left(p_{\text {adj }}=0.0001\right)$ and male-male dyads $\left(p_{\text {adj }}=0.06\right)$. There was no significant difference in compatibility between male-male and male-female dyads $\left(p_{\text {adj }}=0.45\right)$. Compatibility increased with increasing rank distance, indicating that dyads which were close in the hierarchy were less compatible.

\section{Discussion}

Using traditional methods, we found a three-component model for relationship quality in bonobos, similar to that reported for chimpanzees (Fraser et al., 2008; Koski et al., 2012), although some behavioural variables could not be included in this study. This contradicts an earlier attempt to use PCA to measure relationship quality in bonobos, using less behavioural variables and including only one study group (Jaeggi et al., 2010), but provides further evidence that, even when using different behavioural variables, the three component structure as proposed by Cords \& Aureli (2000) can emerge in 
various species even when social organisation differs (Majolo et al., 2010; Fraser \& Bugnyar, 2011; McFarland \& Majolo, 2011). However, when we used Parallel Analysis to avoid overestimation of the number of factors (Horn, 1965), we only found the first two components of relationship quality. Perhaps the second method can be seen as more conservative and more reliable. Since other studies have not used Parallel Analysis, a direct comparison becomes more difficult. On the other hand, the results of the two PCAs were very similar, with only grooming reciprocity changing from component and counter-intervention did not load on either of the components in the second PCA.

Irrespective of our methods, in bonobos the first component 'Value' is very similar to the one reported for chimpanzees (Fraser et al., 2008; Koski et al., 2012), macaques (Majolo et al., 2010; McFarland \& Majolo, 2011) and ravens (Fraser \& Bugnyar, 2010), suggesting that this factor is consistent across taxa. Our second component, 'Compatibility' included aggression frequency and aggression symmetry. Most studies report aggression frequency in the compatibility factor, but aggression symmetry has not been included in any of the previous studies. Other variables reported in compatibility components include: tolerance to approaches (Fraser et al., 2008; Fraser \& Bugnyar, 2010; Majolo et al., 2010; McFarland \& Majolo, 2011), proximity during feeding (Majolo et al., 2010), support (McFarland \& Majolo, 2011), and counter-intervention (Fraser et al., 2008; Fraser \& Bugnyar, 2010; Koski et al., 2012). In our study 'tolerance to approaches' and 'proximity during feeding' could not be included for practical reasons; 'support' grouped with the first component, as it does in most of the other studies; and 'counterintervention' only had weaker loadings on the compatibility factor in either of the PCAs, if we follow Budaev's advice that minimum loadings should be 0.5 or higher (but see Koski et al., 2012 for the use of minimal loadings of 0.4 or higher). In general, our compatibility component matches closely to that reported in previous studies. In our traditional analysis, using Kaiser's criterion, the third component of relationship quality contained 'grooming symmetry' and 'counter-intervention' and we labelled it, tentatively, as security. However, since the Parallel Analysis indicated this last component was not statistically valid, we suggest the third factor should not be retained, and suggest a more cautious interpretation of this third factor.

In general, the full three-component model, as proposed by Cords \& Aureli (2000), has only been found in chimpanzees (Fraser et al., 2008) and 
ravens (Fraser \& Bugnyar, 2011). Notably the third component, 'security', defined as "the predictability of the partner's response to social interactions" (Cords \& Aureli, 2000), seems to be least consistent across studies, and is not always found or differs greatly between studies. This may be partly due to different behavioural variables entered in the model. In one chimpanzee study, the security component contained a measure of consistency of affiliation and grooming symmetry (Fraser et al., 2008). Grooming symmetry was the only variable in a component labelled 'security' in a study on Barbary macaques (McFarland \& Majolo, 2011), but in Japanese macaques the component labelled as '(in)security' comprised aggression and a measure for grooming variability (Majolo et al., 2010). Furthermore, few studies (including this one) could actually include measures of temporal consistency within a dyad. Finally, these consistency measures may also depend on the length of the observation period. In general, this component is less consistent across studies and labelling seems to be more subjective than in the first two components. As mentioned, the inclusion of the Parallel Analysis suggests that the third factor may also not be statistically valid in our study, and therefore, we recommend that future studies also apply this procedure when studying relationship quality and especially before making generalisations about the three-component structure of relationship quality.

Our review above shows that finding ubiquitous components of relationship quality across species can be challenging. Deciding which behavioural variables are entered in the model will depend on practicalities and observational conditions, but the question remains whether there are universal indicators of relationship quality and whether these can or should be complemented with species-specific indicators of relationship quality. The six studies so far all have some behavioural variables in common but differ in many others. Measures of grooming/preening frequency, proximity and aggression frequency are common variables in all studies to date, and consistently group together on the first two components. Other variables, such as food transfers in chimpanzees (Fraser et al., 2008), or embraces in spider monkeys (Rebecchini et al., 2011), are more species specific and may never or only rarely be observed in the other species. For example in spider monkeys, support in was not observed in sufficient number of dyads and was not included in the analyses (Rebecchini et al., 2011). Finally, there are variables that do not consistently cluster onto the same components, such as 
agonistic support, which in Barbary macaques was found to load on the factor labelled Compatibility (McFarland \& Majolo, 2011), but loaded on the Value component in Japanese macaques (Majolo et al., 2010), ravens (Fraser \& Bugnyar, 2012), chimpanzees (Fraser et al., 2008; Koski et al., 2012) and bonobos (this study). It has been suggested that studying relationships at a dyadic level may not always be appropriate, since relationships are rarely symmetrical and may have different implications for a subordinate member of a dyad compared to a dominant member of the dyad (Majolo et al., 2010; McFarland \& Majolo, 2011). Previous studies in bonobos have shown that certain behaviours, such as grooming, can be fairly asymmetric and can be influenced by dominance status of the receiver (Vervaecke et al., 2000b, c; Stevens et al., 2005b; Jaeggi et al., 2010). In the current study we attempted to include symmetry measures for grooming and for aggression in the dyadic analyses, but found that they always grouped with the frequency variables in the Principal Component Analysis. A future study looking at relationship quality on individual rather than dyadic levels, such as has been done in macaques (Majolo et al., 2010; McFarland \& Majolo, 2011), may also be useful in bonobos, so that the asymmetric nature of relationships can be further investigated. Studying relationship value at a dyadic level has allowed us to investigate in more detail the effects of kinship, sex combination, relationship tenure and similarities in rank and age in a more objective way than was previously possible.

\subsection{Kinship}

Kinship had no significant effect on compatibility, but bonobos that were maternally related were found to have higher relationship value than unrelated dyads. This is in line with expectations of inclusive fitness benefits between related individuals (Silk, 2002, 2007) and this higher value was also found in related dyads in chimpanzees (Fraser et al., 2008; Koski et al., 2012), ravens (Fraser \& Bugnyar, 2010) and macaques (Majolo et al., 2010). Due to small sample size it was not possible to test mother-son and maternal kin relationships separately, and both were included in our factor 'kin'. In general, maternal (half-) siblings in bonobos do not seem to form close bonds (Kano, 1992), so the main effect of kin should be attributed to mother-son dyads. In bonobos, mother-son bonds have been shown to be important, as mothers provide agonistic support to their sons even into adulthood (Stevens et al., 2006; Furuichi, 2011) and can influence the mating success of their adult 
sons (Surbeck et al., 2011) In contrast to other studies in macaques (Widdig, 2007; Schülke et al., 2013), but similar to studies on wild chimpanzees (Langergraber et al., 2007; Mitani, 2009), we did not find strong effects of paternal kin relationships on either component of relationship quality.

\subsection{Sex combination}

In captive bonobo groups, female-female dyads were characterised by high value and compatibility. Unlike in previous studies on social bonding in female bonobos (Parish, 1996), all but one female-female dyads in our study were unrelated, demonstrating that high value of female-female value and compatibility in captive groups need not be a side effect of relatedness and underscoring that kinship is not always required for maintenance of high value relationships (Langergraber et al., 2007; Mitani, 2009; Seyfarth \& Cheney, 2012). These results were not unexpected, since we previously reported higher frequency of support among unrelated female bonobos, even though grooming was never more common among female-female dyads and proximity was not always higher in these dyads (Stevens et al., 2006). By using the composite measure value, we can show that the component value is significantly higher in female-female dyads. In matrilocal macaques, femalefemale dyads also have higher relationship value (Majolo et al., 2010; McFarland \& Majolo, 2011), but studies on chimpanzees found no effect of sex combination on relationship value. This makes bonobos unique in being a female-dispersal species, where higher value is found between unrelated females than between mostly related males. Like in our results on bonobos, the chimpanzee studies showed higher compatibility among female-female dyads (Fraser et al., 2008; Koski et al., 2012) and security was highest in male-female dyads and lowest in male-male dyads (Fraser et al., 2008).

While previous studies have suggested that unrelated female chimpanzees can also form close bonds (Baker \& Smuts, 1994; Langergraber et al., 2009; Lehmann \& Boesch, 2009), our study, and comparisons with relationship quality data of captive chimpanzees (Fraser et al., 2008; Koski et al., 2012), suggests that these bonds may differ between species in the aspect of relationship value, which is high in female-female dyads among bonobos but not chimpanzees. In bonobos it still remains to be studied what the effects of high value and high compatibility female bonds are on individual fitness (cf., Silk, 2007). Female-female coalitions may help individual females to obtain and maintain a higher rank, but fitness effects of dominance have not 
been shown in female bonobos. In chimpanzees, such rank effects have been documented in the wild, and high-ranking females have higher reproductive success (Pusey et al., 1997). Data on the direct link between relationship quality and reproductive success are still missing for chimpanzees and bonobos. In baboons, high-ranking females also tend to mature at an earlier age, grow faster, produce healthier infants, have shorter interbirth intervals, live longer and have higher lifetime fitness than lower ranking females (Silk et al., 2003, 2009, 2010; Cheney et al., 2004), but also direct relations between females with strong social relationships and higher reproductive success are reported (Silk et al., 2003, 2009; Silk, 2007). Such fitness effects may be hard to detect in captive primates, and wild studies on the effects of female bonding and dominance on fitness outcomes will be more appropriate.

For male-female dyads, value was significantly lower than female-female dyads but still higher compared to male-male dyads. This can be partly attributed to the mother-son dyads that were included in this category, but it has also been suggested for wild bonobos that males may gain fitness benefits from maintaining long-term friendships with unrelated females (Hohmann \& Fruth, 2003; Surbeck et al., 2012). Compatibility in male-female dyads was lower than female-female dyads but did not significantly differ from male-male dyads. Indeed aggression is most common between males and from females to males, but much more rare between females or from males to females (Hohmann \& Fruth, 2003). Male-male dyads had lower value than male-female or female-female dyads, and lower compatibility than female-female, but not compared to male-female dyads. Similar patterns of behaviour are observed in the wild, where males rarely groom or support each other (Ihobe, 1992; Kano, 1992), but do show high grooming rates with females (Surbeck et al., 2012) and male-male aggression is most common (Kano, 1992; Hohmann \& Fruth, 2003; Surbeck et al., 2012). This opens intriguing questions why male-male relationships in bonobos seem to be more different from those in chimpanzees. In chimpanzees, participation in male coalitions has been shown to influence male mating success, providing a direct link between social bonding and fitness (Gilby et al., 2013). For bonobos, coalitions between males are uncommon (Kano, 1992; Stevens et al., 2005b) and seem to have no fitness benefits. Future studies could focus more on the nature of male-male relationships in wild and captive conditions. 


\subsection{Rank difference}

Rank distance can have a large influence on affiliative behaviour (de Waal, 1991). Previous studies in bonobos have shown that individuals with similar rank groomed more reciprocally but not more frequently (Vervaecke et al., 2000c). In our study, there was no effect of rank difference and relationship Value. However, relationship Compatibility was influenced by rank difference, indicating that aggression was more frequent and more reciprocal in dyads that had smaller rank differences. Since we did not discriminate between mild and severe forms of aggression, it is possible that individuals close in rank have frequent aggressive encounters that are relatively mild; the threshold to reciprocate aggression may be lower in closely ranked individuals, leading to more reciprocal aggression. Rank distance was not investigated in the studies on relationship quality of chimpanzees (Fraser et al., 2008; Koski et al., 2012), and proved to have no effect on components of relationship quality in Japanese macaques (Majolo et al., 2010) or Barbary macaques (McFarland \& Majolo, 2011). The fact that we included aggression symmetry in our analyses, and that this variable grouped with aggression frequency, may also explain why we found an effect. Previous studies in wild and zoo-housed bonobos have shown that group hierarchies in captive bonobos are fairly steep, and male hierarchies especially are steep and asymmetric (Stevens et al., 2007; Surbeck et al., 2010). This underscores the asymmetric nature of social relationships as suggested by Majolo et al. (2010).

\subsection{Relationship tenure}

We found that dyads with longer relationship tenure had higher value. This effect was also found in chimpanzees, where security also decreased with increasing tenure, which the authors could not explain (Fraser et al., 2008). Compatibility (indicating frequency and symmetry of aggression) was not influenced by relationship tenure in our study, similar to what has been found in chimpanzees (Fraser et al., 2008). The fact that value increased with relationship tenure indicates that it may take some time before high value relationships are established, even though the response of bonobos towards young (female) strangers has been shown to be positive (Idani, 1991; Tan \& Hare, 2013). The reciprocal interchange of grooming for support may act as a functional mechanism to strengthen bonds over time, especially between female bonobos (Vervaecke et al., 2000a, b, c; Stevens et al., 2005b). Further 
studies should compare these interchange mechanisms between chimpanzees and bonobos.

\subsection{Age difference}

We found no significant effects of age difference on either component of relationship quality.

Age effects on relationship quality are rarely found. Fraser et al. (2008) found higher value in dyads of more similar age and explained this using the similarity principle proposed by de Waal \& Luttrel (1986), which states that individuals of similar age might have similar needs and possibilities in a social group. There could, however, be an interaction between age and relationship length or tenure. Fraser et al. (2008) studied one group of chimpanzees, in which no adult chimpanzees were introduced and individuals in the study were often born in the group and grew up together. However, in our sampled group of bonobos, dyads with longer tenure do not necessarily have more similar ages, as there has been a high frequency of individual transfers between groups due to the breeding program, so that some individuals with very similar ages were only recently introduced to one another.

Additionally, not only age differences but the actual age of the individuals in the dyads may be influencing the results. Relationships between adolescent bonobos could differ from relationships between adult bonobos in aspects of value and compatibility.

As this is the first large-scale study on relationship quality in bonobos, repeatability of this model should be tested by including more captive and wild populations. Nonetheless, thus far it seems that the first two components, value and compatibility, are very similar to those reported for chimpanzees. However, at least in captivity, the two species differ in that female-female dyads have high relationship value in bonobos, but not in chimpanzees, while in both species relationship compatibility is higher in female-female dyads. Relationships between unrelated bonobo female are characterised by high relationship value and compatibility, which may be the key to their success in maintaining long term bonds, which seem to strengthen over time, perhaps by mechanisms of reciprocal altruism.

\section{Acknowledgements}

We are very grateful to Brian Hare and Shinya Yamamoto for inviting us to contribute to the IPS Congress 2013 Symposium and this accompany- 
ing Special Issue on 'Bonobos: The newly discovered ape'. We thank the curators and keepers of Planckendael Wild Animal Park; Wuppertal Zoo; Apenheul Primate Park and Twycross Zoo for their kind assistance during data collection in these institutions. We thank two anonymous reviewers for their constructive comments on an earlier version of the manuscript. Sumir Keenan did careful language editing. The CRC is structurally supported by the Flemish Government.

\section{References}

Badrian, A. \& Badrian, N. (1984). Social organization of Pan paniscus in the Lomako Forest, Zaire. - In: The pygmy chimpanzee: evolutionary ecology and behaviour (Susman, R.L., ed.). Plenum Press, New York, NY, p. 325-346.

Baker, K.C. \& Smuts, B.B. (1994). Social relationships of female chimpanzees: diversity between captive social groups. - In: Chimpanzee cultures (Wrangham, R.W., McGrew, W.C., de Waal, F.B.M. \& Heltne, P.G., eds). Harvard University Press, Cambridge, MA, p. $227-242$.

Budaev, S.V. (2010). Using principal components and factor analysis in animal behaviour research: caveats and guidelines. - Ethology 116: 472-480.

Cattell, R.B. (1966). The scree test for the number of factors. - Multivar. Behav. Res. 1: 245-276.

Cheney, D.L., Seyfarth, R.M., Fischer, J., Beehner, J.C., Bergman, T.J., Johnson, S.E., Kitchen, D.M., Palombit, R.A., Rendall, D. \& Silk, J.B. (2004). Factors affecting reproduction and mortality among baboons in the Okavango Delta, Botswana. - Int. J. Primatol. 25: 401-428.

Cords, M. \& Aureli, F. (2000). Reconciliation and relationship qualities. - In: Natural conflict resolution (Aureli, F. \& de Waal, F.B.M., eds). University of California Press, Berkeley, CA, p. 177-198.

de Vries, H., Netto, W.J. \& Hanegraaf, P.L.H. (1993). Matman: a program for the analysis of sociometric matrices and behavioural transition matrices. - Behaviour 125: 157-175.

de Waal, F.B.M. (1978). Join-aggression and protective-aggression among captive Macaca fascicularis. - In: Recent advances in primatology (Chivers, D.J. \& Herbert, J., eds). Academic Press, New York, NY, p. 577-579.

de Waal, F.B.M. (1991). Rank distance as a central feature of rhesus monkey social organization: a sociometric analysis. - Anim. Behav. 41: 383-395.

de Waal, F.B.M. \& Luttrell, L.M. (1986). The similarity principle underlying social bonding among female rhesus monkeys. - Folia Primatol. 46: 215-234.

Feh, C. (1999). Alliances and reproductive success in Camargue stallions. — Anim. Behav. 57: 705-713.

Franklin, S.B., Gibson, D.J., Robertson, P.A., Pohlmann, J.T. \& Fralish, J.S. (1995). Parallel analysis: a method for determining significant principal components. - J. Vegetat. Sci. 6: 99-106. 
Franz, C. (1999). Allogrooming behavior and grooming site preferences in captive bonobos (Pan paniscus): association with female dominance. — Int. J. Primatol. 20: 525-546.

Fraser, O.N. \& Bugnyar, T. (2010). The quality of social relationships in ravens. - Anim. Behav. 79: 927-933.

Fraser, O.N., Schino, G. \& Aureli, F. (2008). Components of relationship quality in chimpanzees. - Ethology 114: 834-843.

Frère, C.H., Krützen, M., Mann, J., Connor, R.C., Bejder, L. \& Sherwin, W.B. (2010). Social and genetic interactions drive fitness variation in a free-living dolphin population. - Proc. Natl. Acad. Sci. USA 107: 19949-19954.

Furuichi, T. (1989). Social interactions and the life history of female Pan paniscus in Wamba, Zaire. — Int. J. Primatol. 10: 173-197.

Furuichi, T. (2011). Female contributions to the peaceful nature of bonobo society. - Evol. Anthropol. 20: 131-142.

Furuichi, T., Idani, G., Ihobe, H., Kuroda, S., Kitamura, K., Mori, A., Enomoto, T., Okayasu, N., Hashimoto, C. \& Kano, T. (1998). Population dynamics of wild bonobos (Pan paniscus) at Wamba. — Int. J. Primatol. 19: 1029-1043.

Gilby, I.C., Brent, L.J.N., Wroblewski, E.E., Rudicell, R.S., Hahn, B.H., Goodall, J. \& Pusey, A.E. (2013). Fitness benefits of coalitionary aggression in male chimpanzees. - Behav. Ecol. Sociobiol. 67: 373-381.

Goodall, J. (1986). The chimpanzees of Gombe: patterns of behavior. - The Belknap Press, Cambridge, MA.

Hinde, R.A. (1976). Interactions, relationships and social structure. - Man 11: 1-17.

Hohmann, G. \& Fruth, B. (2002). Dynamics in social organization of bonobos (Pan paniscus). - In: Behavioural diversity in chimpanzees and bonobos (Boesch, C., Hohmann, G. \& Marchant, L.F., eds). Cambridge University Press, Cambridge, p. 138-150.

Hohmann, G. \& Fruth, B. (2003). Intra- and inter-sexual aggression by bonobos in the context of mating. - Behaviour 140: 1389-1413.

Hohmann, G., Gerloff, U. \& Fruth, B. (1999). Social bonds and genetic tests: kinship, association and affiliation in a community of bonobos (Pan paniscus). - Behaviour 136: 1219-1235.

Horn, J.L. (1965). A rationale and test for the number of factors in factor analysis. - Psychometrika 30: 179-185.

Idani, G. (1995). Function of peering behaviour among bonobos (Pan paniscus) at Wamba, Zaire. - Primates 36: 377-383.

Ihobe, H. (1992). Male-male relationships among wild bonobos (Pan paniscus) at Wamba, Republic of Zaire. - Primates 33: 163-179.

Jaeggi, A.V., Stevens, J.M.G. \& van Schaik, C.P. (2010). Tolerant food sharing and reciprocity is precluded by despotism among bonobos but not chimpanzees. - Am. J. Phys. Anthropol. 143: 41-51.

Kaiser, H.F. (1960). The application of electronic computers to factor analysis. — Ed. Psychol. Meas. 20: 141-151.

Kano, T. (1980). Social behavior of wild pygmy chimpanzees (Pan paniscus) of Wamba: a preliminary report. — J. Hum. Evol. 9: 243-260. 
Kano, T. (1992). The last ape: pygmy chimpanzee behavior and ecology. — Stanford University Press, Stanford, CA.

Kappeler, P.M. \& van Schaik, C.P.M. (2002). Evolution of primate social systems. - Int. J. Primatol. 23: 707-740.

Koski, S.E., de Vries, H., van de Kraats, A. \& Sterck, E.H.M. (2012). Stability and change of social relationship quality in captive chimpanzees. — Int. J. Primatol. 33: 905-921.

Kummer, H. (1978). On the value of social relationships to non-human primates: a heuristic scheme. - Soc. Sci. Inform. 17: 697-705.

Langergraber, K.E., Mitani, J. \& Vigilant, L. (2007). The limited impact of kinship on cooperation in wild chimpanzees. — Proc. Natl. Acad. Sci. USA 104: 7786-7790.

Langergraber, K.E., Mitani, J. \& Vigilant, L. (2009). Kinship and social bonds in female chimpanzees (Pan troglodytes). — Am. J. Primatol. 71: 840-851.

Langergraber, K.E., Prüferc, K., Rowney, C., Boesch, C., Crockford, C., Fawcett, K., Inoue, E., Inoue-Muruyama, M., Mitani, J.C., Muller, M.N., Robbins, M.M., Schubert, G., Stoinski, T.S., Viola, B., Watts, D., Wittig, R.M., Wrangham, R.W., Zuberbühler, K., Pääbo, S. \& Vigilant, L. (2012). Generation times in wild chimpanzees and gorillas suggest earlier divergence times in great ape and human evolution. - Proc. Natl. Acad. Sci. USA 109: 15716-15721.

Langergraber, K.E., Mitani, J.C., Watts, D.P. \& Vigilant, L. (2013). Male-female socio-spatial relationships and reproduction in wild chimpanzees. - Behav. Ecol. Sociobiol. 67: 861873.

Lehmann, J. \& Boesch, C. (2009). Sociality of the dispersing sex: the nature of social bonds in West African female chimpanzees, Pan troglodytes. — Anim. Behav. 77: 377-387.

Majolo, B., Ventura, R. \& Schino, G. (2010). Asymmetry and dimensions of relationship quality in the Japanese macaque. — Int. J. Primatol. 31: 736-750.

Martin, P. \& Bateson, P. (1993). Measuring behaviour: an introductory guide, 2nd edn. Cambridge University Press, Cambridge.

Massen, J.J., Overduin-de Vries, A.M., de Vos-Rouweler, A.J., Spruijt, B.M., Doxiadis, G.G. \& Sterck, E.H. (2012). Male mating tactics in captive rhesus macaques (Macaca mulatta): the influence of dominance, markets, and relationship quality. — Int. J. Primatol. 33: 7392.

McFarland, R. \& Majolo, B. (2011). Exploring the components, asymmetry and distribution of relationship quality in wild barbary macaques (Macaca sylvanus). - Plos One 6: e28826.

Mitani, J.C. (2009). Male chimpanzees form enduring and equitable social bonds. - Anim. Behav. 77: 633-640.

Moscovice, L.R., Fiore, A.D., Crockford, C., Kitchen, D.M., Wittig, R., Seyfarth, R.M. \& Cheney, D.L. (2010). Hedging their bets? Male and female chacma baboons form friendships based on likelihood of paternity. - Anim. Behav. 79: 1007-1015.

O'Connor, B.P. (2000). SPSS and SAS programs for determining the number of components using parallel analysis and velicer's MAP test. - Behav. Res. Methods Instrum. Comput. 32: 396-402.

Parish, A.R. (1996). Female relationships in bonobos (Pan paniscus). — Hum. Nat. 7: 61-96. 
Parish, A.R. \& de Waal, F.B.M. (2000). The other "closest living relative": how bonobos (Pan paniscus) challenge traditional assumptions about females, dominance, intra- and intersexual interactions, and hominid evolution. - Ann. NY Acad. Sci. 907: 97-113.

Pereboom, Z., Stevens, J.M.G. \& Van der Eerden, I. (2011). International studbook for bonobo, Pan paniscus Schwarz 1929. - Royal Zoological Society of Antwerp, Antwerp.

Pusey, A., Williams, J. \& Goodall, J. (1997). The influence of dominance rank on reproductive success of female chimpanzees. - Science 277: 828-831.

Rebecchini, L., Schaffner, C. \& Aureli, F. (2011). Risk is a component of social relationships in spider monkeys. — Ethology 117: 691-699.

Schülke, O., Bhagavatula, J., Vigilant, L. \& Ostner, J. (2010). Social bonds enhance reproductive success in male macaques. - Curr. Biol. 20: 2207-2210.

Schülke, O., Wenzel, S. \& Ostner, J. (2013). Paternal relatedness predicts the strength of social bonds among female rhesus macaques. — Plos One 8: e59189.

Seyfarth, R.M. \& Cheney, D.L. (2012). The evolutionary origins of friendship. - Annu. Rev. Psychol. 63: 153-177.

Silk, J.B. (2007). Social components of fitness in primate groups. — Science 317: 1347-1351.

Silk, J.B., Alberts, S.C. \& Altman, J. (2003). Social bonds of female baboons enhance infant survival. - Science 302: 1231-1234.

Silk, J.B., Beehner, J.C., Berglan, T.J., Crockford, C., Engh, A.L., Moscovice, L.R., Wittig, R.M., Seyfarth, R.M. \& Cheney, D.L. (2009). The benefits of social capital: close social bonds among female baboons enhance offspring survival. — Proc. Roy. Soc. Lond. B: Biol. Sci. 276: 3099-3104.

Silk, J.B., Beehner, J.C., Berglan, T.J., Crockford, C., Engh, A.L., Moscovice, L.R., Wittig, R.M., Seyfarth, R.M. \& Cheney, D.L. (2010). Strong and consistent social bonds enhance the longevity of female baboons. - Curr. Biol. 20: 1359-1361.

Stanford, C.B. (1998). The social behavior of chimpanzees and bonobos. - Curr. Anthropol. 39: 399-420.

Stevens, J.M.G., Vervaecke, H., de Vries, H. \& Van Elsacker, L. (2005a). Peering is not a formal indicator of subordination in bonobos (Pan paniscus). - Am. J. Prim. 65: 255267.

Stevens, J.M.G., Vervaecke, H., de Vries, H. \& Van Elsacker, L. (2005b). The influence of the steepness of dominance hierarchies on reciprocity and interchange in captive groups of bonobos (Pan paniscus). - Behaviour 142: 941-960.

Stevens, J.M.G., Vervaecke, H., de Vries, H. \& Van Elsacker, L. (2006). Social structures in Pan paniscus: testing the female bonding hypothesis. — Primates 47: 210-217.

Stevens, J.M.G., Vervaecke, H., de Vries, H. \& Van Elsacker, L. (2007). Sex differences in the steepness of dominance hierarchies in captive bonobo groups. - Int. J. Primatol. 28: 1417-1430.

Stumpf, R. (2007). Chimpanzees and bonobos: diversity within and between species. - In: Primates in perspective (Campbell, C.J., Fuentes, A., MacKinnon, K.C., Panger, M. \& Bearder, S.K., eds). Oxford University Press, Oxford, p. 321-344. 
Surbeck, M., Mundry, R. \& Hohmann, G. (2011). Mothers matter! Maternal support, dominance status and mating success in male bonobos (Pan paniscus). — Proc. Roy. Soc. Lond. B: Biol. Sci. 278: 590-598.

Surbeck, M., Deschner, T., Schubert, G., Weltring, A. \& Hohmann, G. (2012). Mate competition, testosterone and intersexual relationships in bonobos, Pan paniscus. - Anim. Behav. 85: 659-669.

Tan, J. \& Hare, B. (2013). Bonobos share with strangers. — Plos One 8: e51922.

Vervaecke, H., de Vries, H. \& Van Elsacker, L. (2000a). Dominance and its behavioral measures in a captive group of bonobos (Pan paniscus). — Int. J. Primatol. 21: 47-68.

Vervaecke, H., de Vries, H. \& Van Elsacker, L. (2000b). Function and distribution of coalitions in captive bonobos (Pan paniscus). - Primates 41: 249-265.

Vervaecke, H., de Vries, H. \& Van Elsacker, L. (2000c). The pivotal role of rank in grooming and suport behavior in a captive group of bonobos (Pan paniscus). - Behaviour 137: 1463-1485.

White, F.J. (1988). Party composition and dynamics in Pan paniscus. — Int. J. Primatol. 9: 179-193.

Widdig, A. (2007). Paternal kin discrimination: the evidence and likely mechanisms. — Biol. Rev. 82: 319-334.

Wiszniewski, J., Corrigan, S., Beheregaray, L.B. \& Möller, L.M. (2012). Male reproductive success increases with alliance size in Indo-Pacific bottlenose dolphins (Tursiops aduncus). - J. Anim. Ecol. 81: 423-431.

Zwick, W.R. \& Velicer, W.F. (1986). Comparison of five rules for determining the number of components to retain. — Psychol. Bull. 99: 432-442. 